\title{
Laryngeal Schwannoma and its Management: A Case Report
}

Swati Mishra ${ }^{1}$, KKR Ramalingam²

\begin{abstract}
Laryngeal Schwannomas are rare clinical entities that often present with gradually progressing symptoms of hoarseness of voice, dyspnea, globus sensation, and occasionally with a mass in the neck. This most commonly arise in males during the 5th decade of life and is usually supraglottic in location. They are treated surgically. Surgical excision, when done, completely and meticulously prevents recurrence and helps preserve the normal laryngeal function. Schwannomas are amenable to complete surgical resection, unlike neurofibromas which penetrate the nerve fibers and thus have a higher chance of recurrence and malignant transformation. This article talks about a case of laryngeal schwannoma and its management by surgical excision preceded by awake intubation.

Keywords: Awake intubation, Laryngeal schwannoma, Neurofibroma, Supraglottis.

International Journal of Phonosurgery \& Laryngology (2021): 10.5005/jp-journals-10023-1228
\end{abstract}

\section{INTRODUCTION}

Laryngeal schwannomas are rare clinical presentations, being the more common in females in the age group of 5th-6th decade. This is a case report of a man in his 5th decade who presented with complaints of cough and dyspnea and a supraglottic swelling on examination. The report deals with diagnosis of this as laryngeal schwannoma and its subsequent management and follow-up.

\section{Case Description}

A 54-year-old male presented to our OPD with complaints of cough for the past 4 months along with progressive dyspnea. He gave a history of several previous visits to a pulmonologist to rule out the pulmonary cause of cough, after which he was referred to an otolaryngologist. A detailed history revealed that cough was dry in nature and essentially a kind of reflux as was always preceded with some amount of aspiration of food taken in. Dyspnea was more pronounced in the supine position. The routine ear, nose, throat, and neck examination was normal. An Indirect laryngoscopic examination was performed in outpatient department with a 70-degree rigid endoscope which revealed a supraglottic laryngeal swelling. The swelling was smooth in outline, globular, submucosal seen arising from the right aryepiglottic fold and arytenoid, obstructing the laryngeal inlet and moving with respiration. The above findings were confirmed by videolaryngoscopy (Fig. 1) with a flexible fibreoptic laryngoscope.

The CT neck was done for further evaluation which showed a well-defined, oval-shaped swelling measuring $3 \times 3 \mathrm{~cm}$ arising from the right aryepiglottic fold (Fig. 2). The treatment planned was intraoral surgical excision of the swelling. As the swelling was obstructing the laryngeal inlet it was dangerous to intubate the patient under $G A$, hence an awake intubation was done in this case. After successful intubation and desired anesthetic plane, the patient was placed in supine with head extended and neck flexed position. Suspension microlaryngoscopy was performed and the mass was removed with help of microdebrider and scissors (Fig. 3). Operative site was thoroughly reexamined for any residue. After extubation the vocal cords were evaluated for movement and were found to be normal. The mass was sent for histopathological examination which diagnosed mass to be a schwannoma.

\begin{abstract}
'Department of ENT, GMERS Medical College and Hospital, Ahmedabad, Gujarat, India

${ }^{2}$ Department of ENT, KKR ENT Hospital and Research Institute, Chennai,
\end{abstract} Tamil Nadu, India

Corresponding Author: Swati Mishra, Department of ENT, GMERS Medical College and Hospital, Ahmedabad, Gujarat, India, Phone: +91 7358320816, e-mail:mishra_era@yahoo.com

How to cite this article: Mishra S, Ramalingam KKR. Laryngeal Schwannoma and its Management: A Case Report. Int J Phonosurg Laryngol 2021;11(2):66-68.

Source of support: Nil

Conflict of interest: None

The patient was followed 1 week postoperatively. He was symptomatically better and fibreoptic laryngoscopy revealed normal vocal cords with no residual mass (Fig. 4). The patient was followed up at intervals of 6 months for the next 2 years and showed no sign of recurrence.

\section{Discussion}

Schwannomas were described first as a clinical entities by Verocay. These are benign, slow-growing, encapsulated neurogenic tumors. Of all the schwannomas 25-45\% appear in the head and neck region majority being located in parapharyngeal spaces. Laryngeal schwannomas are rare finding accounting to only $0.1-1.5 \%$ of clinical presentations. ${ }^{1,2}$ The most common location in larynx is the supraglottic region like arytenoids, aryepiglottic fold, false cords, and epiglottis. Rarely vocal cords may also be involved. Subglottic location is very rare and often fatal. ${ }^{3}$ These schwannomas originate from the peripherally located Schwann cells of somatic and sympathetic nerves and extrinsically grow and encircle them. In larynx the most common nerve involved is the internal branch of the superior laryngeal nerve. ${ }^{4}$ Schwannomas are found in both male and female, being the more common in females, and can occur in any age group the most common being 4 th and 5 th decade. ${ }^{5}$ As the location is supraglottic the most common symptoms patient presents are due to mass effect like globus sensation, hoarseness (pseudofixation of vocal cords), dyspnea (mainly on lying down),

() The Author(s). 2021 Open Access This article is distributed under the terms of the Creative Commons Attribution 4.0 International License (https://creativecommons. org/licenses/by-nc/4.0/), which permits unrestricted use, distribution, and non-commercial reproduction in any medium, provided you give appropriate credit to the original author(s) and the source, provide a link to the Creative Commons license, and indicate if changes were made. The Creative Commons Public Domain Dedication waiver (http://creativecommons.org/publicdomain/zero/1.0/) applies to the data made available in this article, unless otherwise stated. 


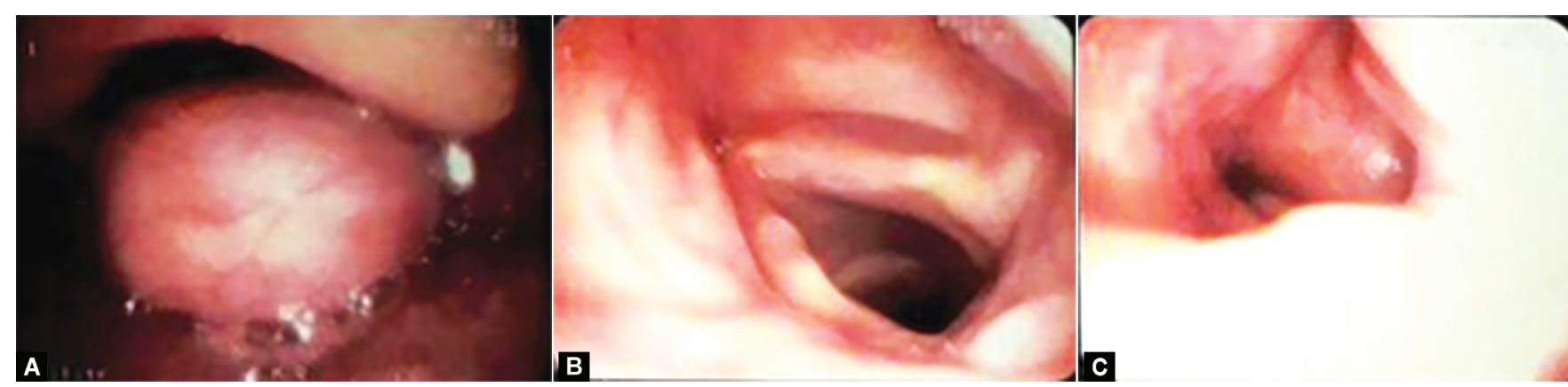

Figs 1 A to C: Videolaryngoscopic view of laryngeal schwannoma. Mass seen arising from right aryepiglottic fold and arytenoids with underneath both vocal cords being normal
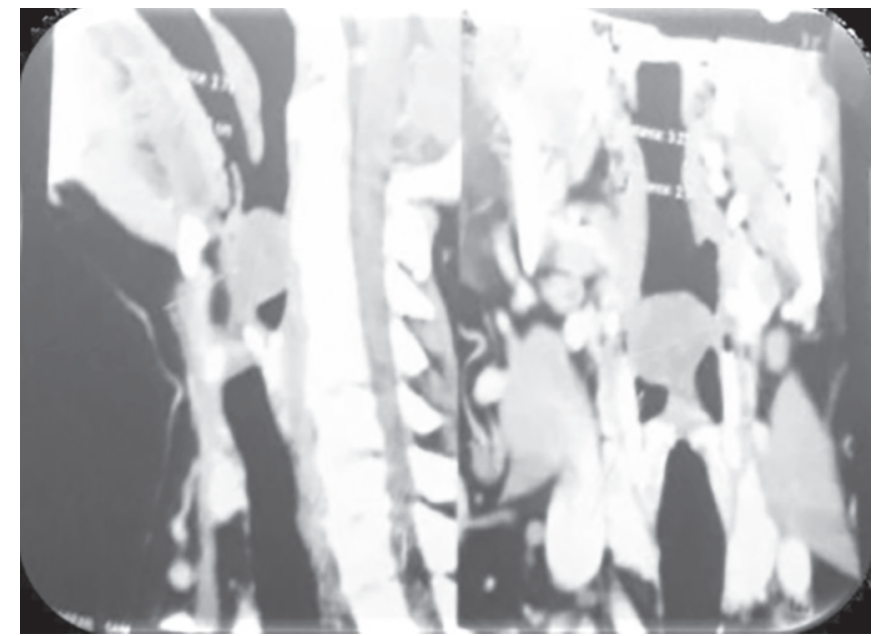

Fig. 2: CT neck shows well-defined oval-shaped, benign nodule measuring $3 \times 3 \mathrm{~cm}$ in right $\mathrm{AE}$ fold

stridor, and dysphagia, and lateral neck mass. In our case one of the chief complaints was reflux cough which indicates the aspiration of food and hence the involvement of the internal branch of the superior laryngeal nerve. Death due to asphyxia has also been reported as a rare symptom. ${ }^{5}$ Schwannomas are slow-growing masses that cause gradual progression of above-mentioned symptoms. ${ }^{6}$

Diagnosis can be made with help of clinical examination by videolaryngoscopy and radiological investigations (X-ray neck, CTscan, and MRI neck). CT and MRI reveal swelling to be smooth and encapsulated. They also entail the exact anatomical location, extension, and the normal anatomical landmark, all of which help in planning the treatment and approach. MRI findings show mass to be smooth hypo to iso-attenuated with muscle on T1w MRI and hyperintense on Tw2. MRI with gadolinium shows hyperintense enhancement of the swelling. ${ }^{7}$ Various differentials which can be deduced from the above findings are neurofibroma, laryngocoele, laryngeal cyst adenomas, chordomas, and malignant tumors. ${ }^{8}$ The definitive diagnosis can be made only by excision and histopathological examination. The histological diagnosis of schwanomma is based on Enger and Weiss criteria. Three characteristic features in the histology are presence of capsule, arrangement of Schwann cells in either Antonia A (Verocay body) or Antoni

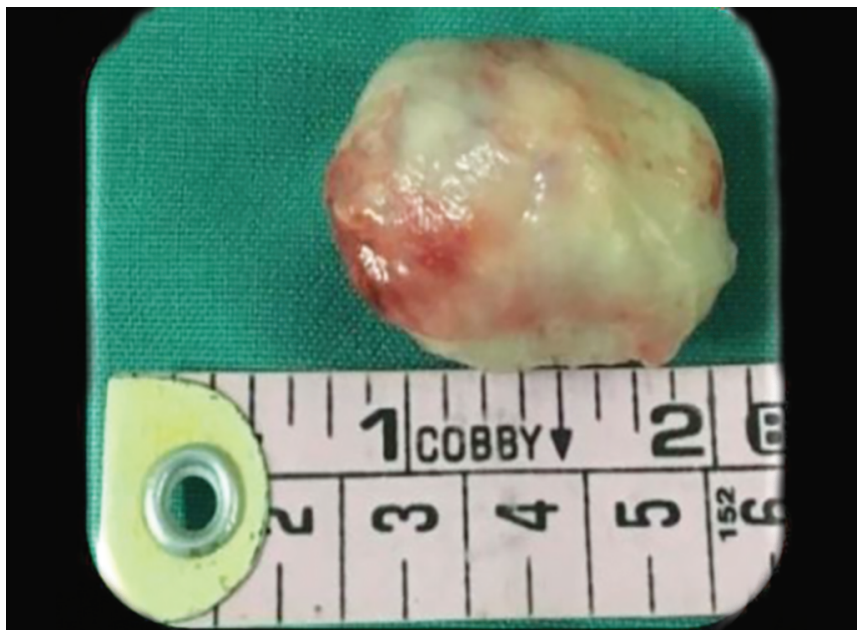

Fig. 3: Excised mass. The mass is smooth globular and homogeneous in appearance

B pattern and S-100 positive staining of Schwann cells. ${ }^{2}$ The most closely resembling swelling is neurofibroma and can be differentiated from schwannoma only by histology. Their differentiation is vital as neurofibromas penetrate the nerve fascicles thus making it difficult for complete surgical excision, unlike schwannoma which only encircle the nerves and can be completely excised. Neurofibroma in contrast to schwannomas has a higher chance of recurrence and malignant transformation. ${ }^{2}$ The definitive treatment of Schwannoma is surgical excision. Depending upon the criticality of the location of the schwannoma, prior airway has to be secured. Various methods of securing airway include awake intubation and sometimes, there is a need for a preoperative tracheostomy. The Schwannomas small in size can be excised via microlaryngeal surgery with either cold instrument or lasers $\left(\mathrm{CO}_{2}\right)$. Transoral robotic-assisted excision has also been mentioned in the literature. ${ }^{9}$ The ones larger in size may require external approaches like a lateral thyrotomy, pharyngectomy, and laryngofissure approaches. ${ }^{6}$ Recurrence of Schwannoma is rare. A meticulous follow-up is necessary for the early recognition of recurrence and its optimum management. Ideally, follow-up is done every 6 months for 2 years followed by yearly for the next 5 years. The recurrence is rare but if encountered; the treatment could be done using laser excision, median thyrotomy, or 


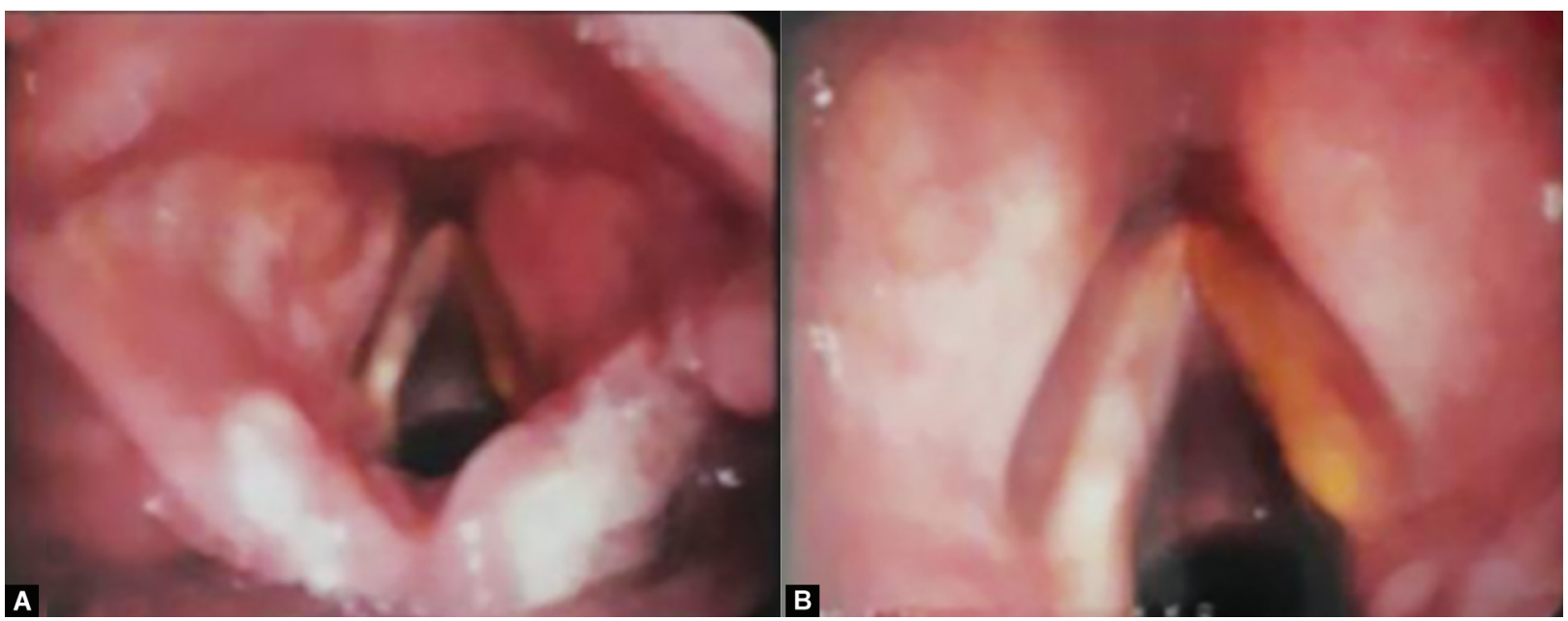

Figs 4A and B: One-week postoperative videolaryngoscopic view of larynx. The operative site is edematous and hemorrhagic without any residual swelling. Bilateral vocal cords were found to be mobile

open approach if the swelling has limited exposure intraorally. Schwannomas rarely have malignant transformation.

\section{Conclusion}

Schwannomas are benign tumors with their laryngeal presentation being a very rare variety. Its rare presentation in the larynx was a point of interest in this case. An additional point that was of the highlight was that in this patient securing airway prior to surgery was challenging, due to its location. Hence, awake intubation was performed in this case.

\section{References}

1. Sanghvi V, Lala M, Borges A, et al. Lateral thyrotomy for neurilemmoma of the larynx. J Laryngol Otol 1999;113(4):346-348. DOI: $10.1017 / \mathrm{s} 0022215100143932$

2. Zbaren $P$, Markwalder R. Schwannoma of the true vocal cord. Otolaryngol Head Neck Surg 1999;121(6):837-839. DOI: 10.1053/hn.1999.v121.a99282
3. Sofi FA, Mir MH, Bagdadi FS, et al. Hidden diagnosis in the subglottic larynx: schwannoma mimicking as bronchial asthma. North Am J Med Sci 2012;4(7):325-327. DOI: 10.4103/1947-2714.98596

4. Cadoni G, Bucci G, Corina L, et al. Schwannoma of the larynx presenting with difficulty in swallowing. Otolaryngol Head Neck Surg 2000;122(5):773-774. DOI: 10.1016/S0194-5998(00)70215-4

5. Gardner PM, Jentzen JM, Komorowski RA, et al. Asphyxial death caused by a laryngeal schwannoma: a case report. J Laryngol Otol 1997;111(12):1171-1173. DOI: 10.1017/s0022215100139635

6. Rosen FS, Pou AM, Quinn JrFB. Obstructive supraglottic schwannoma: a case report and review of the literature. Laryngoscope 2002;112(6):997-1002. DOI: 10.1097/00005537-200206000-00011

7. Park KT, Ahn Y, Kim KH, et al. Schwannoma mimicking laryngocele. Clin Exp Otorhinolaryngol 2010;3(3):166-171. DOI: 10.3342/ ceo.2010.3.3.166

8. Ebmeyer J, Reineke U, Gehl HB, et al. Schwannoma of the larynx. Head Neck Oncol 2009;1:24. DOI: 10.1186/1758-3284-1-24

9. Kayhan FT, Kaya KH, Yilmazbayhan ED. Transoral robotic approach for schwannoma of the larynx.J Craniofac Surg 2011;22(3):1000-1002. DOI: $10.1097 /$ SCS.0b013e3182101580 